\title{
OPEN Microporous hierarchically Zn-MOF as an efficient catalyst for the Hantzsch synthesis of polyhydroquinolines
}

\begin{abstract}
Sayed Mohammad Ramish ${ }^{1}$, Arash Ghorbani-Choghamarani ${ }^{2 \bowtie}$ \& Masoud Mohammadi $^{1}$
A three-dimensional walnut-like $\mathrm{Zn}$-based MOF microsphere system was designed and synthesized via hydrothermal reaction of zinc salt with 4,6-diamino-2-pyrimidinethiol as a tridentate ligand. Besides, $\mathrm{Zn}$ ions were coordinated to the functional groups of the ligand to give a novel $\mathrm{Zn-MOF}$ microsphere material. Afterward, the resultant material was thoroughly characterized using various analysis and physico-chemical methods; including, FT-IR, XRD, TGA, EDX, X-ray mapping, SEM, TEM, and BET analysis. The $\mathrm{Zn}-\mathrm{MOF}$ microspheres were utilized in the Hantzsch reaction for a selective synthesis of asymmetric polyhydroquinolines, using various aromatic aldehydes. Our strategy aims at providing a controlled synthesis of hierarchically nanoporous $\mathrm{Zn}$-MOF microspheres with a welldefined morphology, structure, and excellent catalytic properties. Besides, it would result in having a promising heterogeneous catalyst for a selective synthesis with good yields, short reaction time, a low limit of steric hindrance and electronic effects. Moreover, the heterogeneity of the catalyst is further tested with hot filtration and also the reusability results point.
\end{abstract}

Porous materials with large specific surface areas and other unique properties have gained increasing attention ${ }^{1-3}$. During the last two decades, it has been revealed that Metal-organic frameworks (MOFs) have extensive applications in drugs delivery, absorption and desorption of substances, biomedicines, and catalysis due to their desired morphology, high surface area, stability, active metal sites and controllable pore size ${ }^{4-11}$. In this sense, coordination polymer microspheres have drawn great attention for their potential applications in many fields especially in the catalytic synthesis of various organic and bioorganic molecules via organic transformations ${ }^{12-15}$. They can be regarded as a novel class of crystalline porous compounds formed by the strong bonding of metal ions or clusters to polytopic organic ligands, giving rise to extended networks ${ }^{13,16}$. Since traditional microspheres have some drawbacks, i.e. higher product costs because of expensive excipients, sophisticated equipment and stricter quality control ${ }^{17,18}$, the nanoporous microspheres with low density and high specific surface area have become a pragmatic manner to address the existing problems ${ }^{19-22}$.

Recently, some specific efforts have been made to design effective microsphere catalysts with external pores on the surface or internal pores in the core (which can be regarded as the main difference between porous microspheres and traditional microspheres), instead of using traditional microsphere catalysts based on expensive metals ${ }^{1,12,23-27}$. Besides, porosity is very significant in measuring the capacity-efficiency of the catalysts. Moreover, the applied porous microspheres have been based on the porous structure, i.e. the amount, diameter, the structure of the pores, etc., which can be controlled by chemical and physical techniques ${ }^{28,29}$. Among all of the transition metal-based catalysts used in organic transformations, Zinc has gained great attention due to its precious properties such as low cost, nontoxicity, good availability, and being ecofriendly. Therefore, many studies have shown the implementation of catalytic systems based on $\mathrm{Zn}$ using various linkers in organic reactions ${ }^{30-35}$.

The asymmetric reaction of an aldehyde, two components of $\beta$-keto esters, and a nitrogen donor is known as Hantzsch method which generates chiral dihydropyridines. Besides, they have been regarded as useful precursors to many compounds of biological and pharmaceutical interest, i.e. different natural products ${ }^{36,37}$. The application and synthetic methods for polyhydroquinolines synthesis have previously been reviewed ${ }^{36}$. When transition metal catalysts are present, polyhydroquinolines with the functionalized aromatic rings can be generated from this reaction ${ }^{38-41}$. Among various catalysts developed so far, the efficiency of the catalytic systems is generally

${ }^{1}$ Department of Chemistry, Faculty of Science, Ilam University, P. O. BOX. 69315516, Ilam, Iran. ${ }^{2}$ Department of Organic Chemistry, Faculty of Chemistry, Bu-Ali Sina University, 6517838683 Hamedan, Iran. ${ }^{\square}$ email: a.ghorbani@basu.ac.ir 
<smiles>Nc1cc(N)nc(S)n1</smiles>

Scheme 1. The synthesis of $\mathrm{Zn}-\mathrm{MOF}$ microspheres.

limited by their low yield, use of volatile organic solvents, harsh reaction condition, aggregation tendency as well as poor mechanical properties, high catalyst loading, tedious workup procedure, and separation of catalyst residue from the reaction mixture ${ }^{36,42}$. Although this method is practically useful for the synthesis of interesting pharmaceutical products, its selectivity and substrate scope still need to be improved ${ }^{36,43,44}$. We propose to develop a novel selective heterogeneous catalytic system for the recognition of the polyhydroquinoline derivatives to facilitate the screening of the catalysts for this asymmetric reaction.

In this work, we studied the hydrothermal synthesis of novel Zn-based nanoporous microspheres, by the reaction of zinc nitrate hexahydrate salt with 4,6-Diamino-2-pyrimidinethiol containing amine, thiol, and pyrimidine functional groups. We also found out that $\mathrm{Zn}-\mathrm{MOF}$ can be used as a rapid catalyst to synthesize the polyhydroquinolines with excellent yields. It represents a new method for a clean and rapid synthesis of asymmetric dihydropyridines. Herein, these results are reported.

\section{Experimental}

Materials. All reagents and solvents were purchased from Merck and used without additional purification.

Synthesis of nanoporous Zn-MOF microspheres. To prepare Zn-MOF porous microspheres, 4,6-Diamino-2-pyrimidinethiol ( $1 \mathrm{mmol})$ was dissolved in water $(2 \mathrm{~mL})$ and, then, it was added to a solution of DMF $(12 \mathrm{~mL})$ containing $2 \mathrm{mmol}$ of $\mathrm{Zn}\left(\mathrm{NO}_{3}\right)_{2} \cdot 6 \mathrm{H}_{2} \mathrm{O}$ salt through 20 minutes of stirring at $80^{\circ} \mathrm{C}$ until reaching a clear solution. Afterward, the white suspension was transferred into a Teflon lined autoclave reactor and heated at $160{ }^{\circ} \mathrm{C}$ for $24 \mathrm{~h}$ (Scheme 1), which was then cooled to the room temperature at a gradient of $40{ }^{\circ} \mathrm{C}$ per hour. Subsequently, the target $\mathrm{Zn}$-MOF microsphere yellow powder was obtained after the sonication in ethyl acetate.

General procedure for the catalytic synthesis of polyhydroquinolines. A mixture of the substituted aromatic aldehyde $(1.0 \mathrm{mmol})$, ethyl acetoacetate $(1 \mathrm{mmol})$ and dimedone $(1 \mathrm{mmol})$ was stirred with Ammonium acetate - as a green $\mathrm{NH}_{3}$ source $(1.2 \mathrm{mmol})$ - and Zn-MOF microspheres $(8 \mathrm{mg})$ in PEG-400 $(2 \mathrm{~mL})$ for $70-180 \mathrm{~min}$ at $80^{\circ} \mathrm{C}$. Completion of the following reaction has been analyzed via TLC. Subsequent to cooling at room temperature, the catalyst was separated using simple filtration. Besides, the resultant mixture was extracted using Ethyl acetate and water derivation to gather unrefined polyhydroquinoline product, which was further purified through recrystallization in ethanol.

Selected spectral data. Ethyl-4-(4-hydroxyphenyl)-2,7,7-trimethyl-5-oxo-1,4,5,6,7,8-hexahydroquinoline-3-carboxylate. $\quad{ }^{1} \mathrm{H}$ NMR (500 MHz, DMSO-d6): $\delta(\mathrm{ppm}) 0.85(\mathrm{~s}, 3 \mathrm{H}), 1.00(\mathrm{~s}, 3 \mathrm{H}), 1.12(\mathrm{t}, \mathrm{J}=7.2 \mathrm{~Hz}, 3 \mathrm{H})$, $1.95(\mathrm{~d}, \mathrm{~J}=16.0 \mathrm{~Hz}, 1 \mathrm{H}), 2.14(\mathrm{~d}, \mathrm{~J}=16.0 \mathrm{~Hz}, 1 \mathrm{H}), 2.22-2.30(\mathrm{~m}, 4 \mathrm{H}), 2.40(\mathrm{~d}, \mathrm{~J}=16.8 \mathrm{~Hz}, 1 \mathrm{H}), 3.97(\mathrm{q}, \mathrm{J}=7.2 \mathrm{~Hz}$, 2H), $4.74(\mathrm{~s}, 1 \mathrm{H}), 6.54(\mathrm{~d}, \mathrm{~J}=8.4 \mathrm{~Hz}, 2 \mathrm{H}), 6.93(\mathrm{~d}, \mathrm{~J}=8.0 \mathrm{~Hz}, 2 \mathrm{H}), 8.94(\mathrm{~s}, 1 \mathrm{H}), 9.01(\mathrm{~s}, 1 \mathrm{H})$; (Fig. S1); ${ }^{13} \mathrm{C}$ NMR (126 MHz, DMSO) $\delta 194.3,167.1,155.3,149.2,144.4,138.5,128.4,114.6,114.4,110.4,104.1,59.0,34.8,32.2$, 29.2, 26.5, 18.3, 14.2 (Fig. S2).

Ethyl-4-(2-nitrophenyl)-2,7,7-trimethyl-5-oxo-1,4,5,6,7,8-hexahydroquinoline-3-carboxylate $\quad(4 i) . \quad{ }^{1} \mathrm{H} \quad \mathrm{NMR}$ (500 MHz, DMSO): $\delta(\mathrm{ppm}) 0.76(\mathrm{~s}, 3 \mathrm{H}), 0.93-1.11(\mathrm{~m}, 6 \mathrm{H}), 1.89(\mathrm{~d}, \mathrm{~J}=16.0 \mathrm{~Hz}, 1 \mathrm{H}), 2.15(\mathrm{~d}, \mathrm{~J}=16.0 \mathrm{~Hz}, 1 \mathrm{H})$, $2.21-2.29(\mathrm{~m}, 4 \mathrm{H}), 2.39(\mathrm{~d}, \mathrm{~J}=17.2 \mathrm{~Hz}, 1 \mathrm{H}), 3.82-3.96(\mathrm{~m}, 2 \mathrm{H}), 5.65(\mathrm{~s}, 1 \mathrm{H}), 7.29(\mathrm{t}, \mathrm{J}=8.0 \mathrm{~Hz}, 1 \mathrm{H}), 7.42(\mathrm{~d}$, $\mathrm{J}=8.0 \mathrm{~Hz}, 1 \mathrm{H}), 7.55(\mathrm{~m}, 1 \mathrm{H}), 7.72(\mathrm{~m}, 1 \mathrm{H}), 9.12(\mathrm{~s}, 1 \mathrm{H})$; (Fig. S3). ${ }^{13} \mathrm{C}$ NMR $(126 \mathrm{MHz}, \mathrm{DMSO}) \delta 194.0,166.6$, $149.8,147.7,146.0,142.0,130.7,126.9,123.7,123.5,109.9,103.1,59.1,32.1,28.9,26.3,18.3,13.9$ (Fig. S4).

Ethyl-4-(3-nitrophenyl)-2,7,7-trimethyl-5-oxo-1,4,5,6,7,8-hexahydroquinoline-3-carboxylate. $\quad{ }^{1} \mathrm{H} \quad$ NMR (500 MHz, DMSOd6): $\delta(\mathrm{ppm}) 0.84(\mathrm{~s}, 3 \mathrm{H}), 1.02(\mathrm{~s}, 3 \mathrm{H}), 1.12(\mathrm{t}, \mathrm{J}=6.8 \mathrm{~Hz}, 3 \mathrm{H}), 1.97(\mathrm{~d}, \mathrm{~J}=16.0 \mathrm{~Hz}, 1 \mathrm{H}), 2.16(\mathrm{~d}$, $\mathrm{J}=16.4 \mathrm{~Hz}, 1 \mathrm{H}), 2.27-2.35(\mathrm{~m}, 4 \mathrm{H}), 2.46(\mathrm{t}, \mathrm{J}=16.8 \mathrm{~Hz}, 1 \mathrm{H}), 3.97(\mathrm{q}, \mathrm{J}=7.2 \mathrm{~Hz}, 2 \mathrm{H}), 4.97(\mathrm{~s}, 1 \mathrm{H}), 7.50(\mathrm{~m}, 1 \mathrm{H})$, $7.61(\mathrm{~m}, 1 \mathrm{H}), 7.97-7.99(\mathrm{~m}, 2 \mathrm{H}), 9.24(\mathrm{~s}, 1 \mathrm{H})$; (Fig. S5). ${ }^{13} \mathrm{C}$ NMR $(126 \mathrm{MHz}$, DMSO) $\delta 194.4,166.5,166.4,150.2$, $149.8,147.4$, 146.4, 146.2, 134.47, 122.0, 120.9, 109.3, 102.7, 101.1, 59.3, 36.5, 32.2, 29.1, 26.3, 18.4, 14.0 (Fig. S6).

\section{Results and discussions}

This study reports the synthesis of nanoporous Zn-MOF microspheres to develop an efficient and novel heterogeneous catalyst to synthesize polyhydroquinolines as an important dihydropyridine-containing scaffold via Hantzsch reaction. The resulting samples were characterized using various techniques.

Synthesis and characterization of the catalyst. The heterogeneous $\mathrm{Zn}-\mathrm{MOF}$ microsphere catalyst was successfully prepared by hydrothermal reaction of $\mathrm{Zn}\left(\mathrm{NO}_{3}\right)_{2} \cdot 6 \mathrm{H}_{2} \mathrm{O}$ and 4,6-Diamino-2-pyrimidinethiol, using the deprotonation of amine and thiol groups. Afterwards, metal coordination bonding to the $\mathrm{Zn}$ ions was 


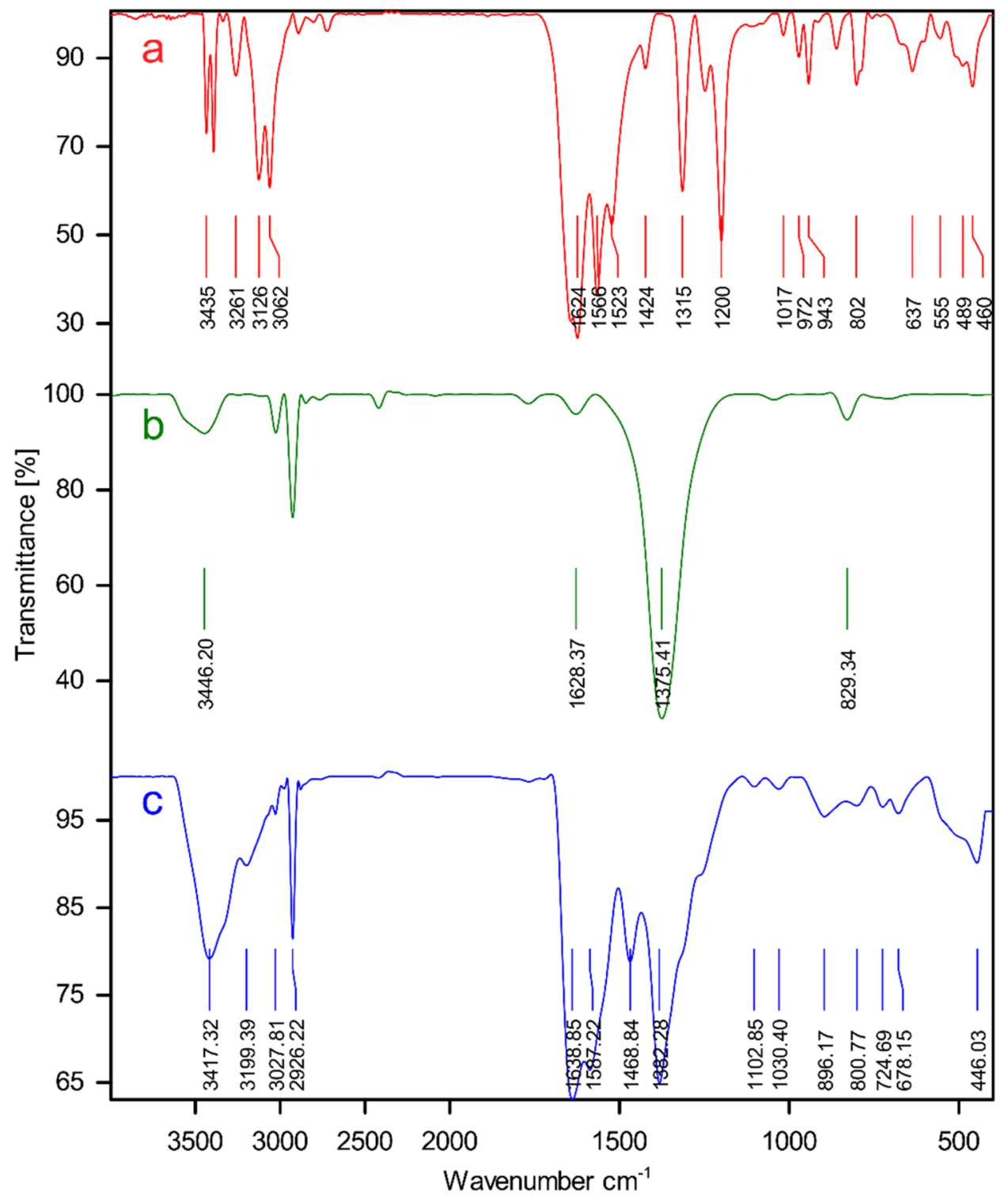

Figure 1. FT-IR Spectrums of (a) 4,6-Diamino-2-pyrimidinethiol, (b) $\mathrm{Zn}\left(\mathrm{NO}_{3}\right)_{2} \cdot 6 \mathrm{H}_{2} \mathrm{O}$ and (c) nanoporous Zn-MOF microspheres.

made to give a novel $\mathrm{Zn}$-based microsphere catalyst. This newly synthesized nanoporous microsphere catalyst was fully characterized using various techniques; including, FT-IR, XRD, TGA, EDX, X-ray mapping, SEM, TEM and BET analyses. The results obtained from these techniques confirmed the successful preparation of this novel catalyst.

Structural and chemical composition analysis. The changes in the chemical features during the synthesis of the composite were measured by FT-IR spectroscopy. Figure 1 shows the FT-IR spectra of (a) 4,6-Diamino2-pyrimidinethiol, (b) $\mathrm{Zn}\left(\mathrm{NO}_{3}\right)_{2} \cdot 6 \mathrm{H}_{2} \mathrm{O}$ and (c) nanoporous $\mathrm{Zn}$-MOF microspheres, respectively. Regarding the FT-IR spectrum of the 4,6-Diamino-2-pyrimidinethiol (Fig. 1a), the bands at $3339 \mathrm{~cm}^{-1}$ and $3435 \mathrm{~cm}^{-1}$ are attributed to the stretching vibration of $\mathrm{N}-\mathrm{H}$ bonds of the free $\mathrm{NH}_{2}$ groups while those at $1523 \mathrm{~cm}^{-1}$ are related to the $\mathrm{C}=\mathrm{C}$ group of the pyrimidine ring. The Amine bands disappeared in the FT-IR spectra of Zn-MOF microspheres (Fig. 1c), indicating that the $\mathrm{NH}_{2}$ groups have been deprotonated and coordinated to the $\mathrm{Zn}$ atoms. Moreover, the bending vibration of $\mathrm{NH}_{2}$ at $1638 \mathrm{~cm}^{-1}$ in the $\mathrm{Zn}-\mathrm{MOF}$ microspheres shows a shift towards higher frequencies as compared to the primary sample. This shift can be assigned to the presence of $\mathrm{Zn}$ ions in MOF structure and the reduction of the single bond nature of the amine and thiol groups, which confirms the successful complexion of $\mathrm{Zn}$ ions with the $\mathrm{N}$ and $\mathrm{S}$ atoms in the framework. The strong $\mathrm{C}=\mathrm{C}$ stretching vibration band at $1468 \mathrm{~cm}^{-1}$ and $\mathrm{C}-\mathrm{N}$ stretch band at $1102 \mathrm{~cm}^{-1}$ provide shreds of evidence confirming the successful synthesis of the final MOF (Fig. 1c). 


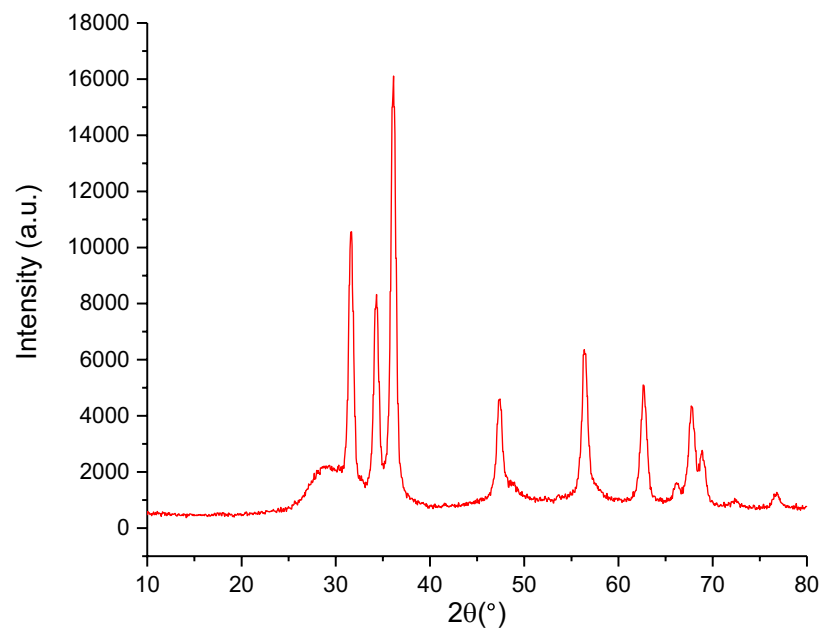

Figure 2. XRD pattern of nanoporous $\mathrm{Zn}-\mathrm{MOF}$ microspheres.

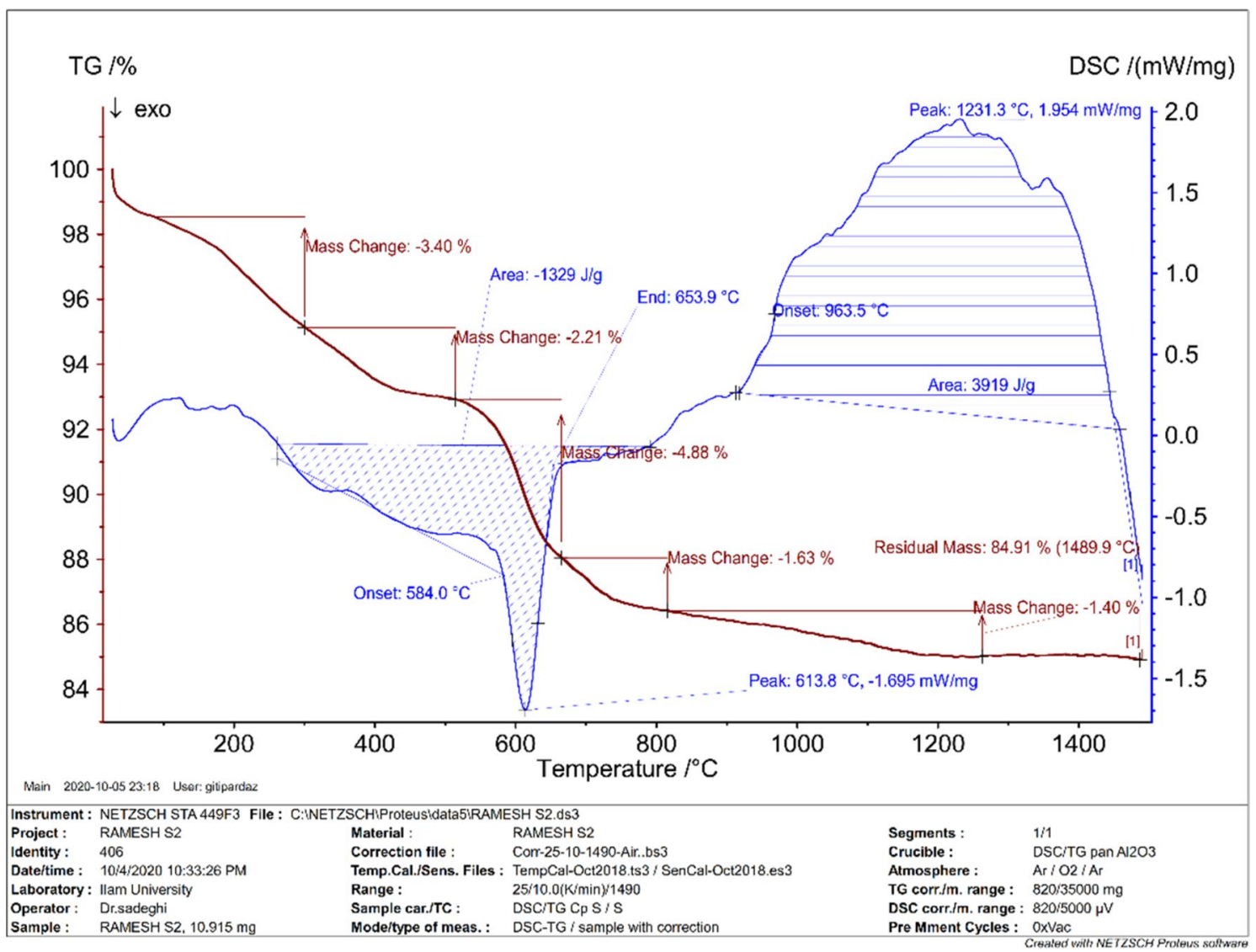

Figure 3. TGA (Maroon line) and DSC (Blue line) curves of nanoporous Zn-MOF microspheres.

The crystalline phases of nanoporous Zn-MOF microspheres were examined by Powder X-ray diffraction (PXRD) analysis as shown in Fig. 2. The characteristic diffraction peaks corresponding to the obtained framework are found at $2 \theta=31.62^{\circ}, 34.49^{\circ}, 36.15^{\circ}, 47.42^{\circ}, 56.3^{\circ}, 62.57^{\circ}, 67.75^{\circ}, 68.95^{\circ}, 72.51^{\circ}$ and $76.82^{\circ}$ which effectively coincide with simulated XRD patterns, showing that the structure agrees with the literature reports $\mathrm{s}^{7,45,46}$. Based on XRD results, we can also observe that the Zn-MOF microspheres obtained from zinc nitrate show sharp characteristic peaks, suggesting the high crystalline nature of the obtained nanoporous $\mathrm{Zn}$-MOF microspheres.

To estimate the mass ratios and the thermal stability of nanoporous Zn-MOF microspheres, the TGA-DSC technique was employed. Figure 3 represents TGA (Maroon line) and DSC (Blue line) curves of Zn-MOF microspheres at the temperature range of $25-1500^{\circ} \mathrm{C}$. Regarding the TGA curve, the weight loss which was occurred below $200^{\circ} \mathrm{C}$ was attributed to the release of the physically adsorbed moisture, water and organic solvents from 


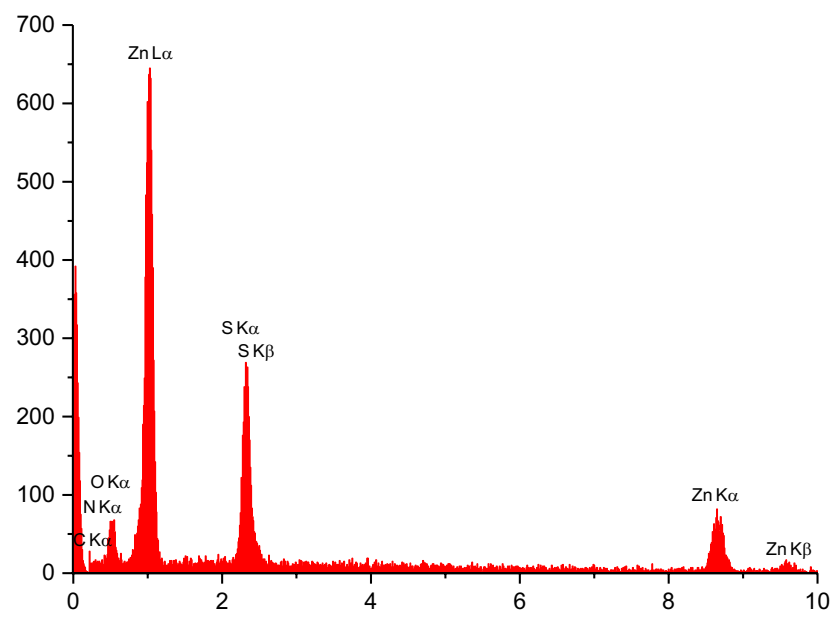

Figure 4. EDX Analysis of nanoporous Zn-MOF microspheres.
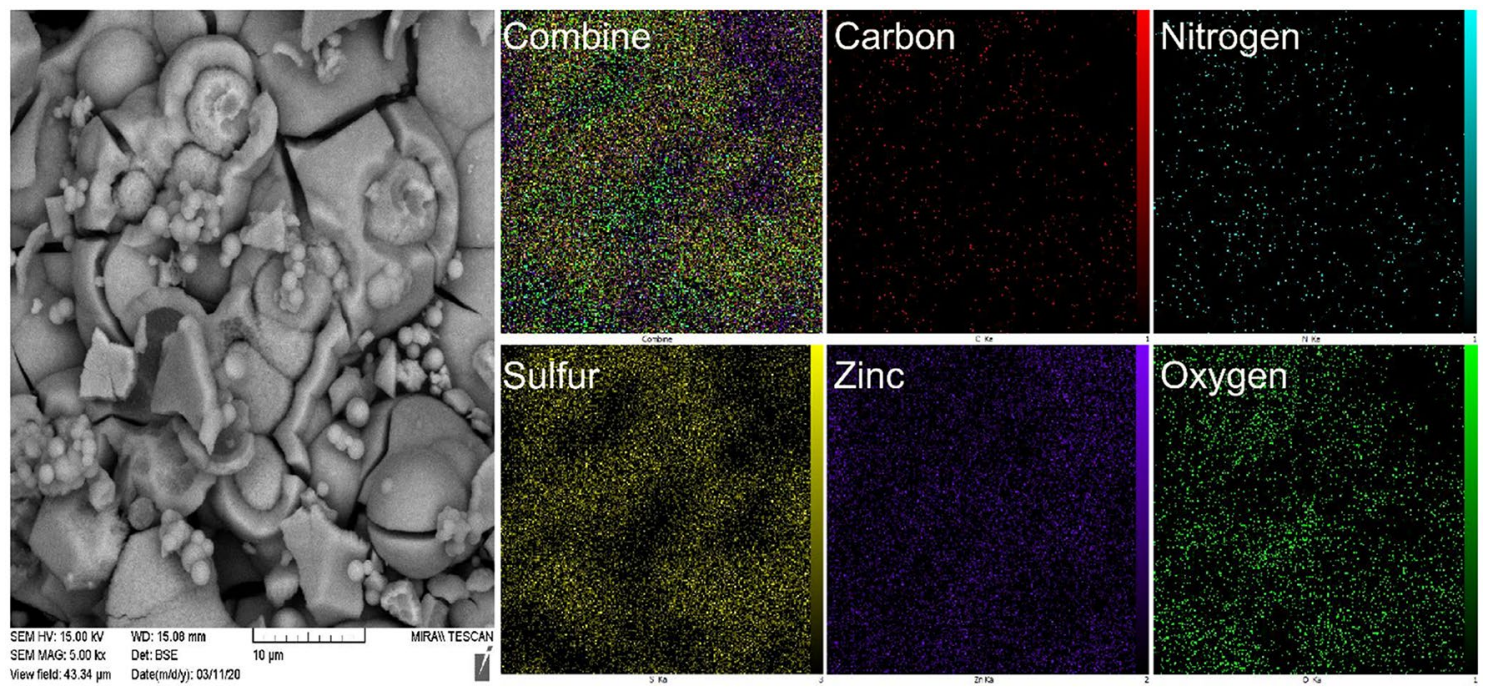

Figure 5. X-ray mapping analysis of Zn-CA-CPS.

the sample ${ }^{47}$. The next weight loss $(10.61 \%)$ in the region of $200-800{ }^{\circ} \mathrm{C}$ can be associated with the decomposition of 4,6-Diamino-2-pyrimidinethiol ligand ${ }^{48,49}$. In addition, the weight loss at $450^{\circ} \mathrm{C}$ indicates the decomposition of the framework. A basic point about the nature of the $\mathrm{Zn}-\mathrm{MOF}$ catalyst is the thermal stability of the catalyst up to $200^{\circ} \mathrm{C}$. Therefore, this catalyst can be used under various catalytic reaction conditions up to $200{ }^{\circ} \mathrm{C}$.

As shown in Fig. 4, energy dispersive X-ray (EDX) analysis was applied to determine the chemical composition of nanoporous $\mathrm{Zn}-\mathrm{MOF}$ microspheres. The results indicate the presence of $53.25 \mathrm{~W} \%$ of $\mathrm{Zn}$ species in the obtained nanoporous $\mathrm{Zn}-\mathrm{MOF}$ microsphere catalyst. In addition, the presence of carbon (10.70 W\%), nitrogen (9.11 W\%), sulfur (13.97 W\%) and oxygen (12.97 W\%) elements in the prepared nanoporous material was also confirmed by these measurements. These observations support the high purity of the prepared Zn-MOF microspheres.

The uniform distribution of the index elements ( $, S, N, O$, and $\mathrm{Zn}$ ) of the obtained nanoporous material is observed at the X-ray mapping analysis (Fig. 5). In addition, the uniform distribution of the Zn element shows that it has evenly coordinated to $\mathrm{N}$ and $\mathrm{S}$ elements, indicating the presence of ligand in the obtained frameworks. Besides, the uniform incorporation of the activated $\mathrm{Zn}$ catalytic species with $\mathrm{S}$ and $\mathrm{N}$ groups shows that an excellent catalytic surface has been formed.

The surface morphology of the prepared nanoporous material was assessed by scanning electron microscopy (FE-SEM) and transmission electron microscopy (TEM) analyses. The FE-SEM images for pure nanoporous Zn-MOF microspheres are presented in Fig. 6. A closer look at the SEM images of the obtained nanoporous material indicates that the $\mathrm{Zn}$-MOF has been formed in a uniform, three-dimensional, walnut-like and hierarchically nanoporous microparticle morphology, which can be attributed to a proper bonding in-between the metal and linker. Moreover, the SEM images show that these Zn-MOF microparticles are composed of a collection of nanoparticles having a mean particle size of $23-27 \mathrm{~nm}$. The results indicate that the Zn-MOF microspheres 

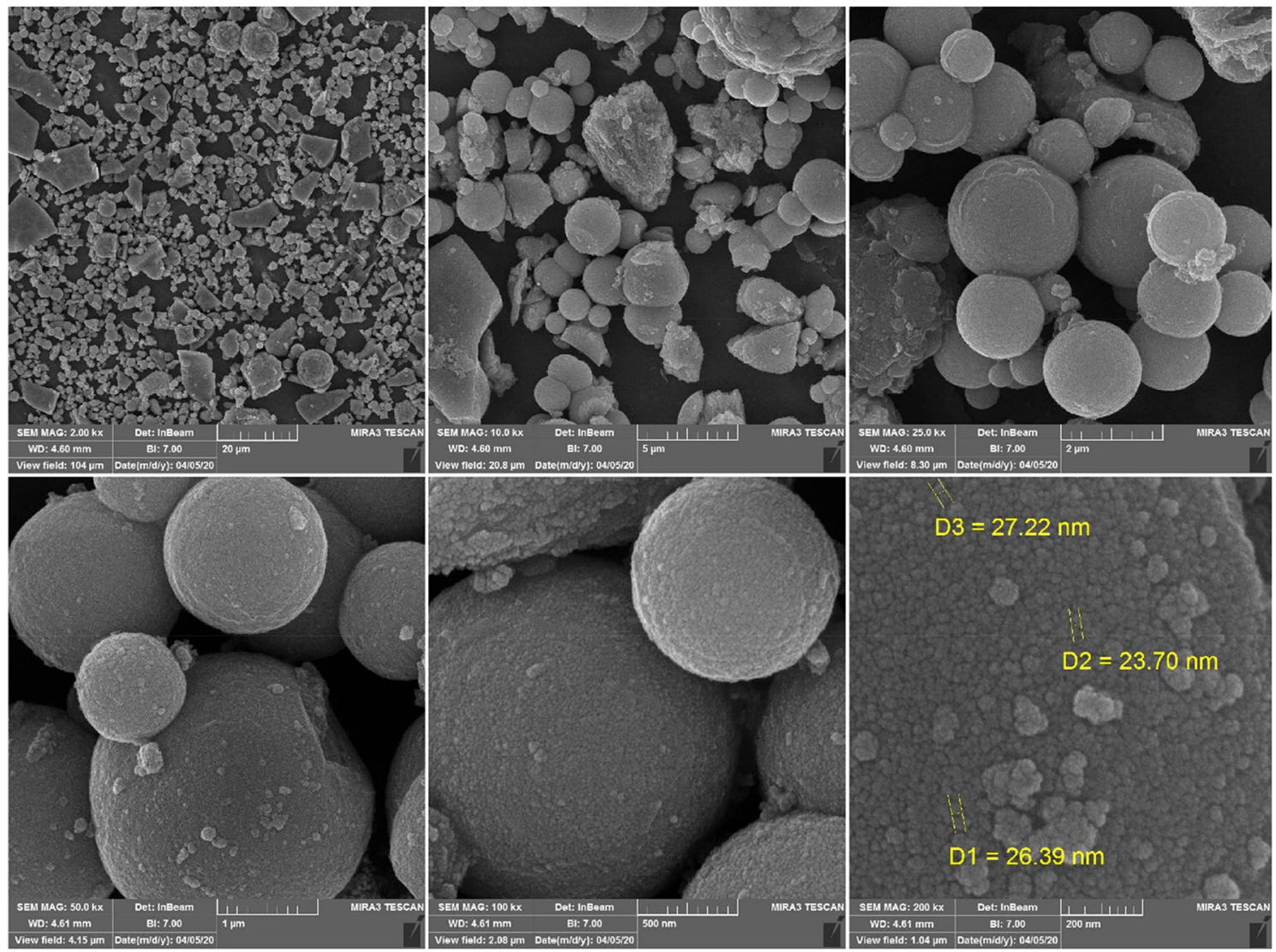

Figure 6. SEM images of three-dimensional walnut-like, hierarchically nanoporous Zn-MOF microspheres.

have been formed in a uniform manner. Besides, there is no indication of agglomeration of the particles which results in optimizing the catalytic properties of the samples.

By applying the transmission electron microscopy technique, high quality images from the synthesized crystalline nanoporous Zn-MOF microspheres were obtained (Fig. 7). Evidently, the synthesized Zn-MOF microspheres showed characteristics of crystalline spherical structure, referring to the fact that the optioned material is a solid sphere. Particle size distribution histogram of the as-prepared microspheres gave distribution dimensions across a range from 90 to $170 \mathrm{~nm}$, with an average size of $130 \mathrm{~nm}$ (Fig. 7).

The porosity and surface characteristics of nanoporous $\mathrm{Zn}-\mathrm{MOF}$ microspheres were explored by the $\mathrm{N}_{2}$ adsorption/desorption analysis. The obtained results are illustrated in Fig. 8. Accordingly, Zn-MOF microspheres exhibited a Brunauer-Emmett-Teller (BET) surface area of $63.33 \mathrm{~m}^{2} \mathrm{~g}^{-1}$. Besides, the pore volumes and pore size distribution of nanoporous $\mathrm{Zn}-\mathrm{MOF}$ microspheres are calculated by $\mathrm{BJH}$ analysis, as $0.088 \mathrm{~cm}^{3} \mathrm{~g}^{-1}$ and $1.22 \mathrm{~nm}$, belonging to the range of micropores materials.

\section{Catalytic study}

After the successful characterization of the synthesized Zn-MOF microsphere, we applied these nanoporous materials as a novel catalyst for the multicomponent synthesis of polyhydroquinolines under diverse conditions (Table 1). Moreover, we optimized conditions of the reaction for Hantzsch synthesis of dihydropyridine scaffolds using para-chlorobenzaldehyde, dimedone, ethyl acetoacetate, and ammonium acetate as the model reaction. Afterwards, various parameters of the reaction, including the amount of catalyst, solvent and temperature, were evaluated for the model reaction, the results of which are summarized in Table 1 . When there is no catalyst in the reaction, no reaction takes place (Table 1, entry 1). In this sense, it is worth mentioning that the existence of $\mathrm{Zn}-\mathrm{MOF}$ microsphere is required for this type of Hantzsch reaction. The reaction proceeds faster by increasing the catalyst amount up to $10 \mathrm{mg}$. According to the results, $10 \mathrm{mg}$ of the catalyst is required for the reaction. Besides, using smaller amounts of the catalyst will cause the reaction to be incomplete (Table 1, entry 4 \& 5). Increasing the amount of the catalyst by more than $10 \mathrm{mg}$ does not affect the efficiency percentage (Table 1, entry 7). Additionally, the catalytic effect of 4,6-diamino-2-pyrimidinethiol and $\mathrm{Zn}\left(\mathrm{NO}_{3}\right)_{2} \cdot 6 \mathrm{H}_{2} \mathrm{O}$ was investigated on the model reaction. It was observed that they cannot efficiently catalyze the reaction and, as a result, the product is obtained in low yields in $85 \mathrm{~min}$. Among various solvents used in the reaction, the results indicate that the PEG-400 which showed a higher efficiency, as compared to all solvents tested with $96 \%$ of isolated yield, may serve as both the solvent and the phase transfer catalyst in this type of reaction (Table 1, entry 6). Finally, the reaction was performed at different temperatures and, significantly, we observed that low temperature continued 

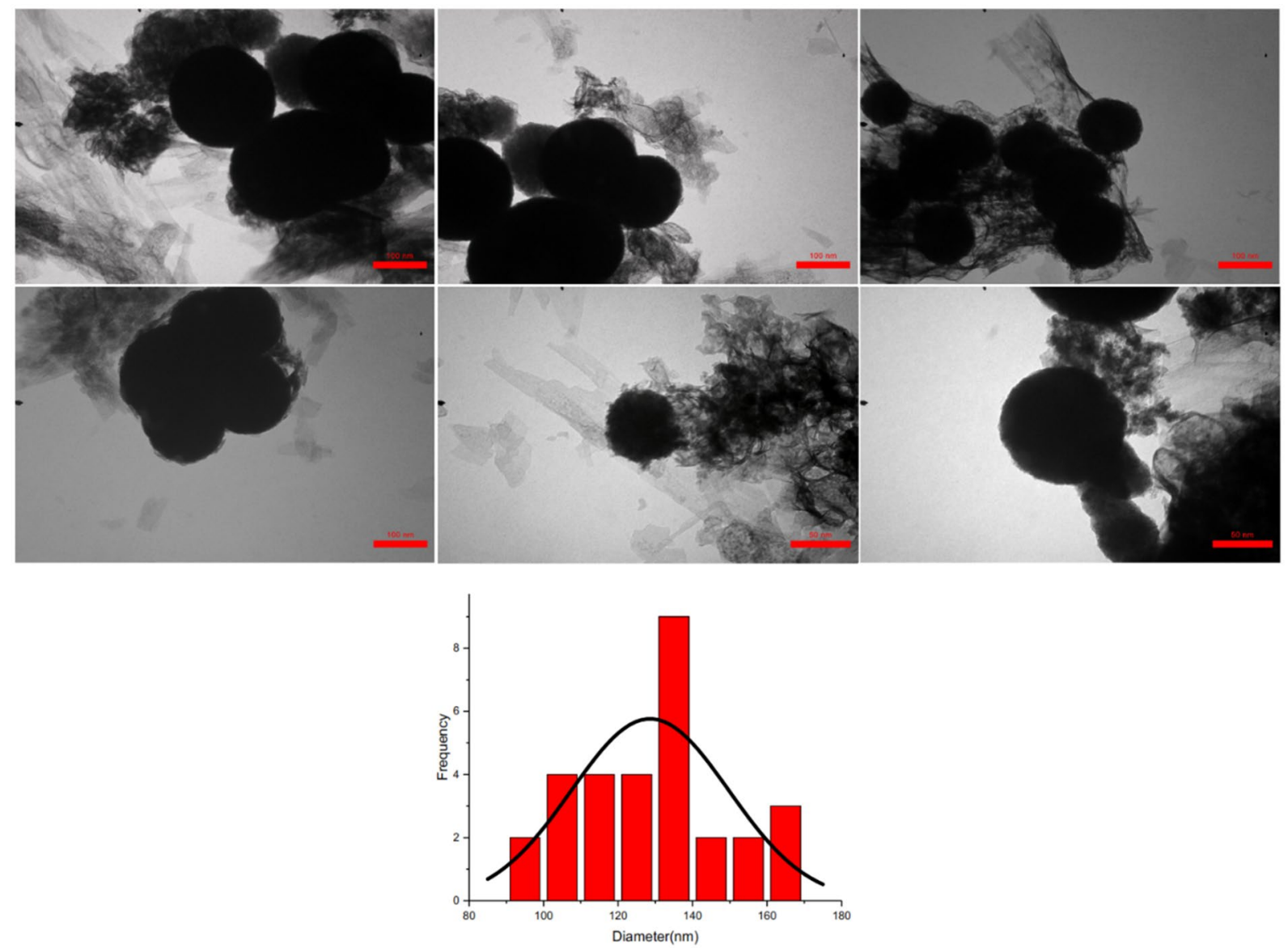

Figure 7. TEM images and particle size distribution histogram of Zn-MOF microspheres.

with lower efficiency (Table 1, entry 6,12-14). Regarding the optimization studies, the optimum conditions for this reaction are $10 \mathrm{mg}$ of $\mathrm{Zn}-\mathrm{MOF}$ in the PEG- 400 at $80^{\circ} \mathrm{C}$ (Table 1 , entry 6).

After optimizing the reaction conditions, we explored the scope of the reaction with various electron-donating and electron-withdrawing groups of aldehydes. In all cases, the products were made in high yields (Table 2). Although the results indicate that ortho and/or meta-substituted aryl aldehydes react slowly, as compared to the para isomers, electron-releasing and electron-withdrawing groups give an excellent yield of the product.

It is worth noting that an appropriate mechanism for the synthesis reaction of polyhydroquinoline derivatives, catalyzed by nanoporous $\mathrm{Zn}-\mathrm{MOF}$ microspheres, was proposed (Scheme 2$)^{36}$. Firstly, the reaction was granted to start through deprotonation of the dimedone which underwent Knoevenagel condensation reaction with an aldehyde to form an $\alpha, \beta$-unsaturated compound. Subsequently, the Michael addition of imine which was achieved from the combination of $\mathrm{NH}_{3}$ with ethyl acetoacetate on the $\alpha, \beta$-unsaturated carbonyl compounds was followed by cyclization reaction, generating a six-membered ring. Finally, the dehydration gave the final polyhydroquinoline products (Scheme 2$)^{36}$.

\section{Catalyst reusability studies}

Recyclability of the heterogeneous catalyst put an end to the use of harmful and costly metal catalysts while decreasing the cost of products. These factors are of crucial importance from an economical point of view. Recyclability of the nanoporous Zn-MOF microsphere was investigated in the model reaction. The catalyst was separated after completion of the reaction, washed with ethyl acetate and acetone and, then, dried at $70{ }^{\circ} \mathrm{C}$. The dried catalyst was reused for the next cycle. Moreover, the recycled catalyst was employed in the four sequential cycles; the yield of the reaction was decreased moderately after the fourth run of the reaction, as illustrated in Fig. 9. Furthermore, we aimed at investigating the FT-IR spectrum of the reused microporous Zn-MOF catalyst (Fig. 10). Evidently, the stability of as-prepared catalyst is confirmed due to the fact that, almost all of the characteristic peaks are the same as the fresh catalyst.

\section{Hot filtration}

The hot filtration test was another analysis to approve the heterogeneous nature of the nanoporous $\mathrm{Zn}-\mathrm{MOF}$ microspheres in the Hantzsch synthesis of polyhydroquinolines. On this basis, the model reaction was studied again under the optimized reaction condition. After $43 \min$ (59\% conversion), the Zn-MOF was removed from the reaction by simple filtration. Afterwards, the rest of the reaction was stirred in the absence of the catalyst 

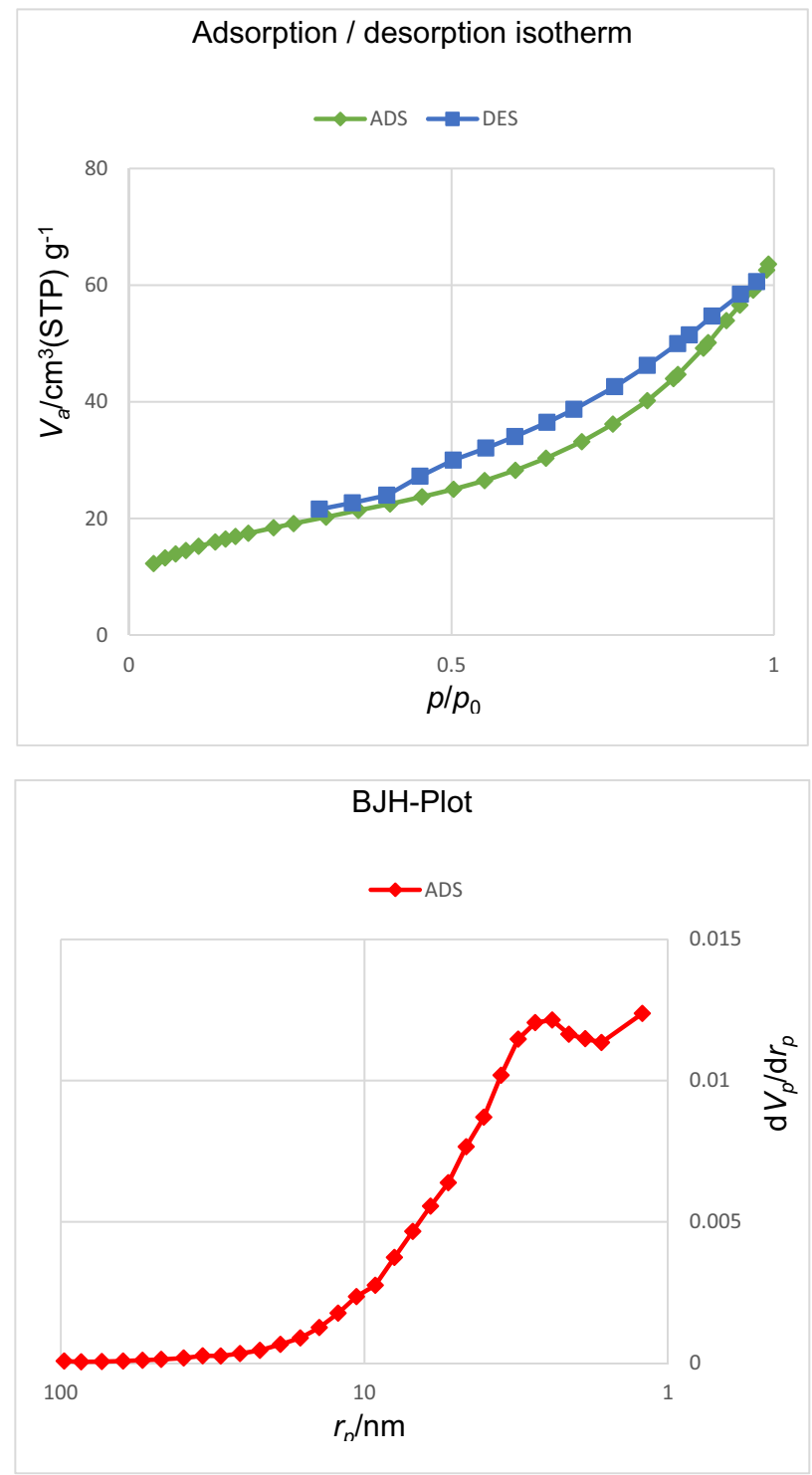

Figure 8. $\mathrm{N}_{2}$ adsorption/desorption isotherms and $\mathrm{BJH}$-Plot of the nanoporous $\mathrm{Zn}$-MOF microspheres.

for a further $43 \mathrm{~min}$. The obtained results show that the $\mathrm{Zn}$-based MOF played a catalytic role in the reaction without the $\mathrm{Zn}$ leaching into the solution or framework degradation.

\section{Comparison}

In the last part of our studies, to demonstrate the profit of nanoporous $\mathrm{Zn}-\mathrm{MOF}$ microspheres as a heterogeneous catalyst in Hantzsch reaction, our resultant and reaction conditions were compared with those of the reported acid, base and metal catalysts in the synthesis of polyhydroquinolines (Table 3). As depicted in Table 3, the nanoporous $\mathrm{Zn}-\mathrm{MOF}$ microspheres are the most efficient catalysts to synthesize polyhydroquinolines. Significantly, most of the reported methods toil from the absence of commonness for the condensation reactions of the deactivated aldehydes. In addition, the reported synthetic paths have some limitations, such as requiring extreme temperature or long duration, large amounts of the catalyst and, most importantly, the use of hazardous solvents to give excellent yields. Promising results obtained in the presence of $\mathrm{Zn}$-MOF microspheres should be ascribed to the nanoporous structure of the catalyst. The two roles of PEG- 400 (solvent and phase transfer catalyst) avouch the better contact between the catalyst and the reactants and, thus, substantially raising the catalytic activity and stability.

\section{Conclusion}

In conclusion, we have reported an efficient and novel three-dimensional walnut-like, microporous $\mathrm{Zn}-\mathrm{MOF}$ microsphere catalytic system by hydrothermal method. Significantly, we were able to accelerate the asymmetric Hantzsch reaction with various aryl aldehydes including electron-donor and electron-acceptor groups for in situ production of polyhydroquinolines. The present protocol gives an excellent yield of the product within a 


\begin{tabular}{|c|c|c|c|c|c|c|}
\hline Entry & Catalyst & Amount Catalyst (mg) & Solvent & Temperature $\left({ }^{\circ} \mathrm{C}\right)$ & Time (min) & Yield $(\%)^{\mathrm{a}, \mathrm{b}}$ \\
\hline 1 & - & - & PEG-400 & 80 & 85 & 30 \\
\hline 2 & 4,6-Diamino-2-pyrimidinethiol & 10 & PEG-400 & 80 & 85 & 41 \\
\hline 3 & $\mathrm{Zn}\left(\mathrm{NO}_{3}\right)_{2} \cdot 6 \mathrm{H}_{2} \mathrm{O}$ & 10 & PEG-400 & 80 & 85 & 30 \\
\hline 4 & Zn-MOF & 5 & PEG-400 & 80 & 85 & 75 \\
\hline 5 & Zn-MOF & 6 & PEG-400 & 80 & 85 & 79 \\
\hline 6 & Zn-MOF & 10 & PEG-400 & 80 & 85 & 96 \\
\hline 7 & Zn-MOF & 12 & PEG-400 & 80 & 85 & 92 \\
\hline 8 & Zn-MOF & 10 & EtOH & 80 & 195 & 83 \\
\hline 9 & Zn-MOF & 10 & $\mathrm{H}_{2} \mathrm{O}$ & 80 & 120 & N.R \\
\hline 10 & Zn-MOF & 10 & DMF & 80 & 210 & 60 \\
\hline 11 & Zn-MOF & 10 & DMSO & 80 & 95 & 70 \\
\hline 12 & $\mathrm{Zn}-\mathrm{MOF}$ & 10 & PEG-400 & 25 & 120 & N.R \\
\hline 13 & Zn-MOF & 10 & PEG-400 & 60 & 85 & 21 \\
\hline 14 & $\mathrm{Zn}-\mathrm{MOF}$ & 10 & PEG-400 & 100 & 85 & 44 \\
\hline
\end{tabular}

Table 1. Optimization of the reaction conditions for the Hantzsch condensation of para-Chlorobenzaldehyde, dimedone, ethyl acetoacetate, and ammonium acetate as the model reaction for the synthesis of polyhydroquinolines. ${ }^{a}$ Isolated yield. ${ }^{b}$ Reaction conditions: 4 -Chlorobenzaldehyde $(1 \mathrm{mmol})$, dimedone $(1 \mathrm{mmol})$, ethyl acetoacetate $(1 \mathrm{mmol})$, ammonium acetate $(1.2 \mathrm{mmol})$, catalyst $(\mathrm{mg})$ and solvent $(2 \mathrm{~mL})$. 


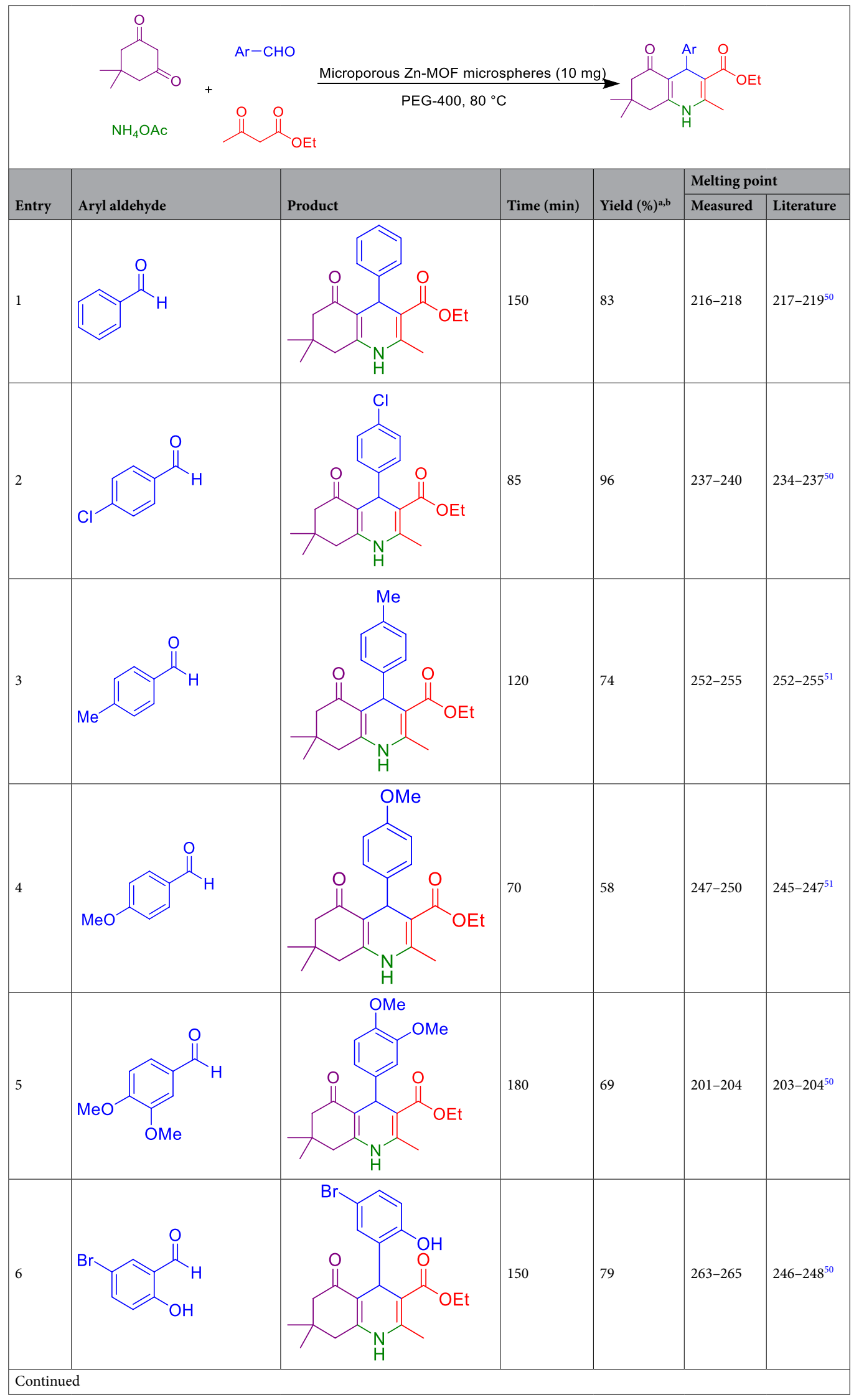




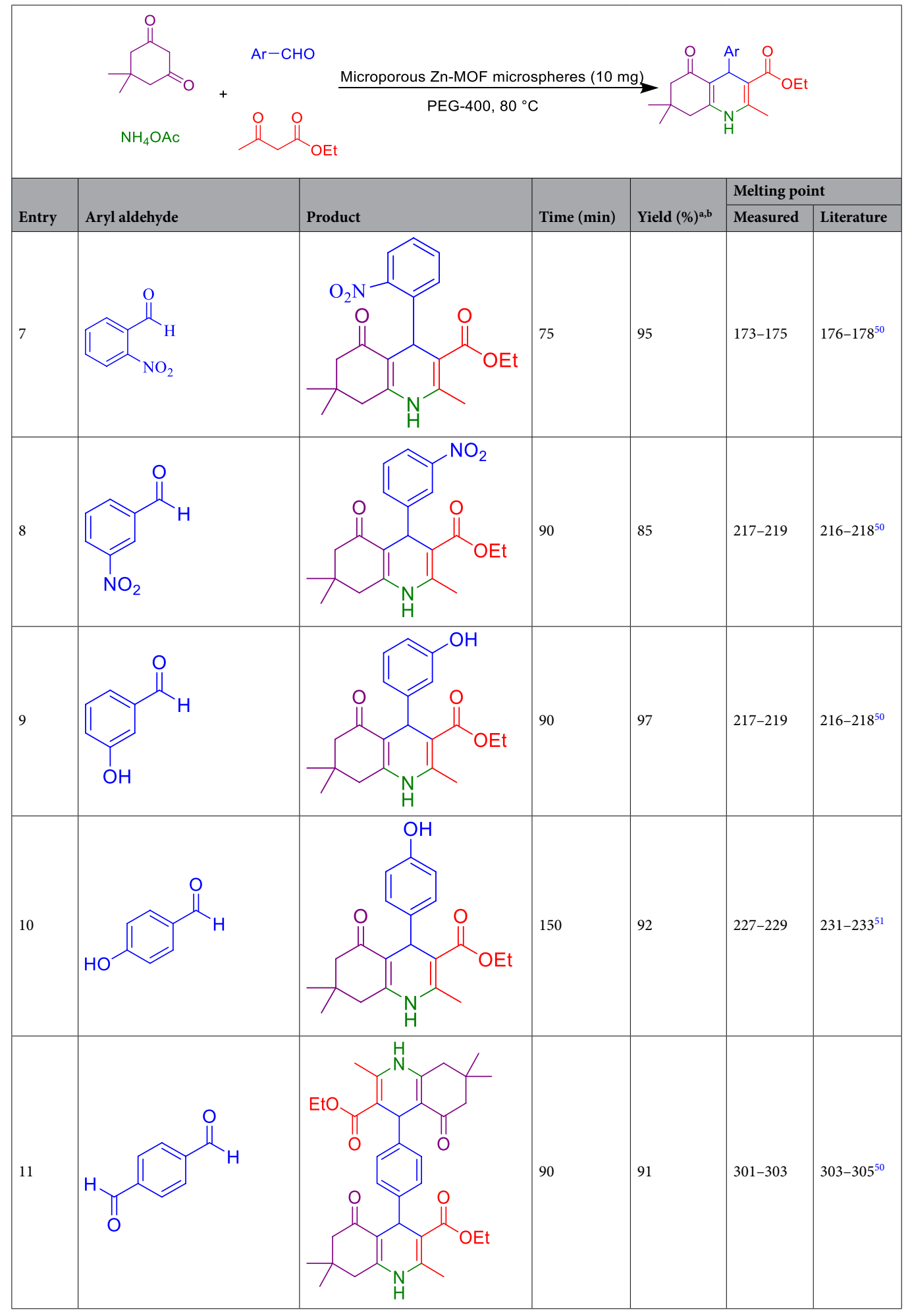

Table 2. Hantzsch synthesis of polyhydroquinoline derivatives in the presence of Zn-MOF in PEG-400 at $80{ }^{\circ} \mathrm{C}$. ${ }^{a}$ Isolated yields. ${ }^{b}$ Reaction conditions: Aromatic aldehyde $(1 \mathrm{mmol})$, dimedone $(1 \mathrm{mmol})$, ethyl acetoacetate $(1 \mathrm{mmol})$, ammonium acetate $(1.2 \mathrm{mmol}), \mathrm{Zn}-\mathrm{MOF}(10 \mathrm{mg})$ and PEG-400 $(2 \mathrm{~mL})$ at $80^{\circ} \mathrm{C}$. ${ }^{\mathrm{c}}$ Reaction conditions: Aromatic aldehyde $(1 \mathrm{mmol})$, dimedone $(2 \mathrm{mmol})$, ethyl acetoacetate $(2 \mathrm{mmol})$, ammonium acetate $(2.4 \mathrm{mmol}), \mathrm{Zn}-\mathrm{MOF}(20 \mathrm{mg})$ and PEG-400 $(4 \mathrm{~mL})$ at $80^{\circ} \mathrm{C}$. 


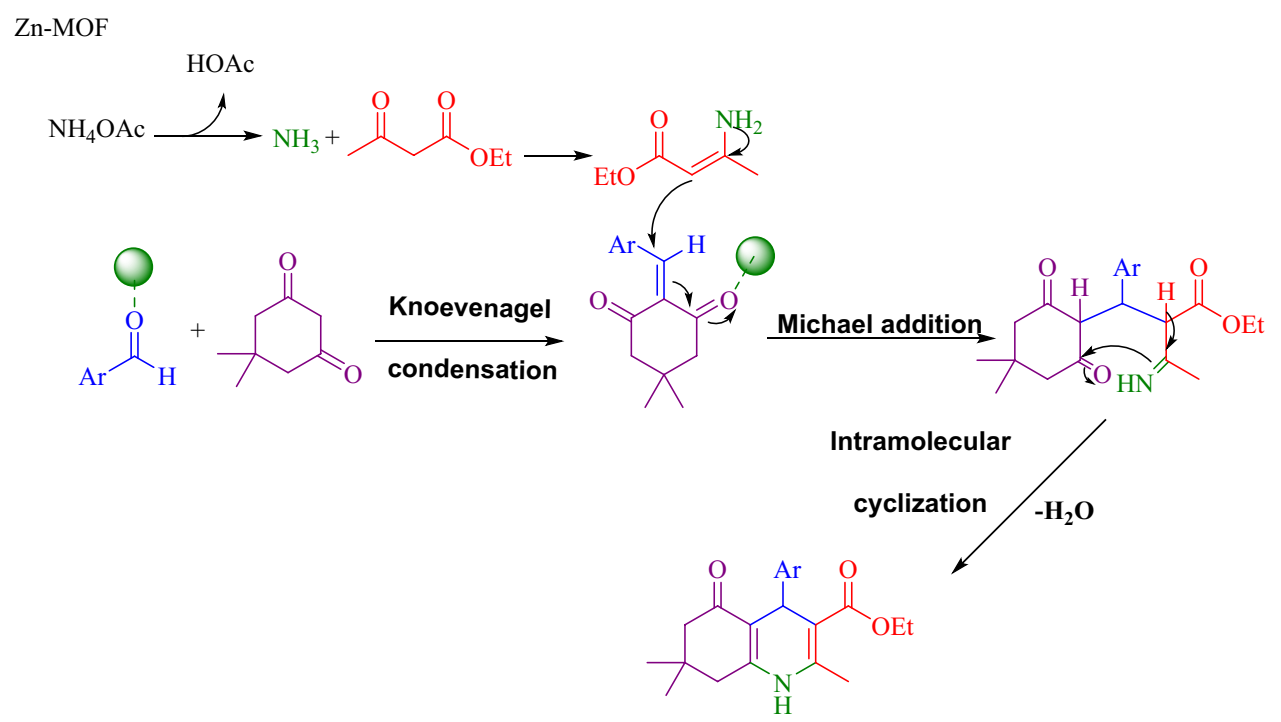

Scheme 2. Proposed mechanism for the synthesis of polyhydroquinolines in the presence of nanoporous Zn-MOF microspheres.

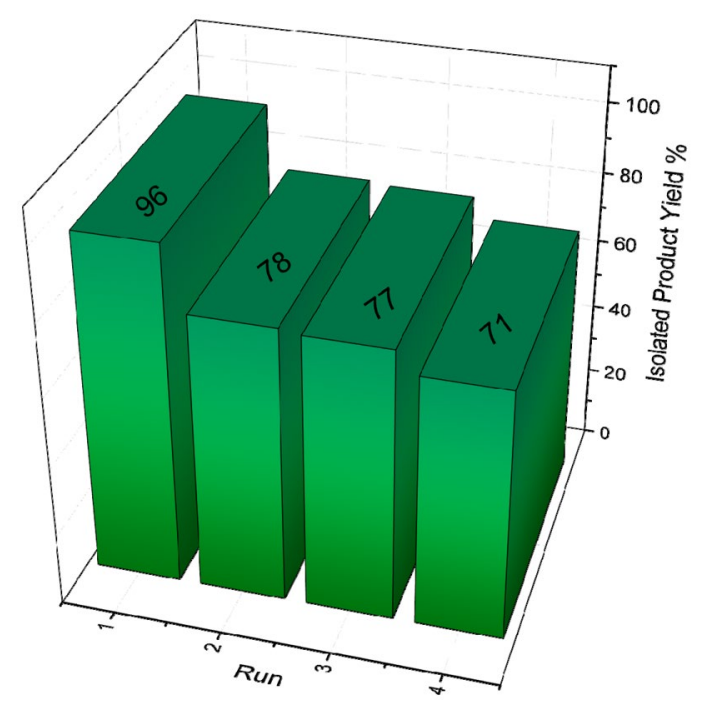

Figure 9. Recyclability of the nanoporous Zn-MOF microspheres.

short reaction time, by an easy workup and no need for further purification of the product. The novelty, simple synthesis procedure, no use of harmful solvents, facile filtration, high yield and, especially, reusability are some of the advantages of the developed catalyst. 


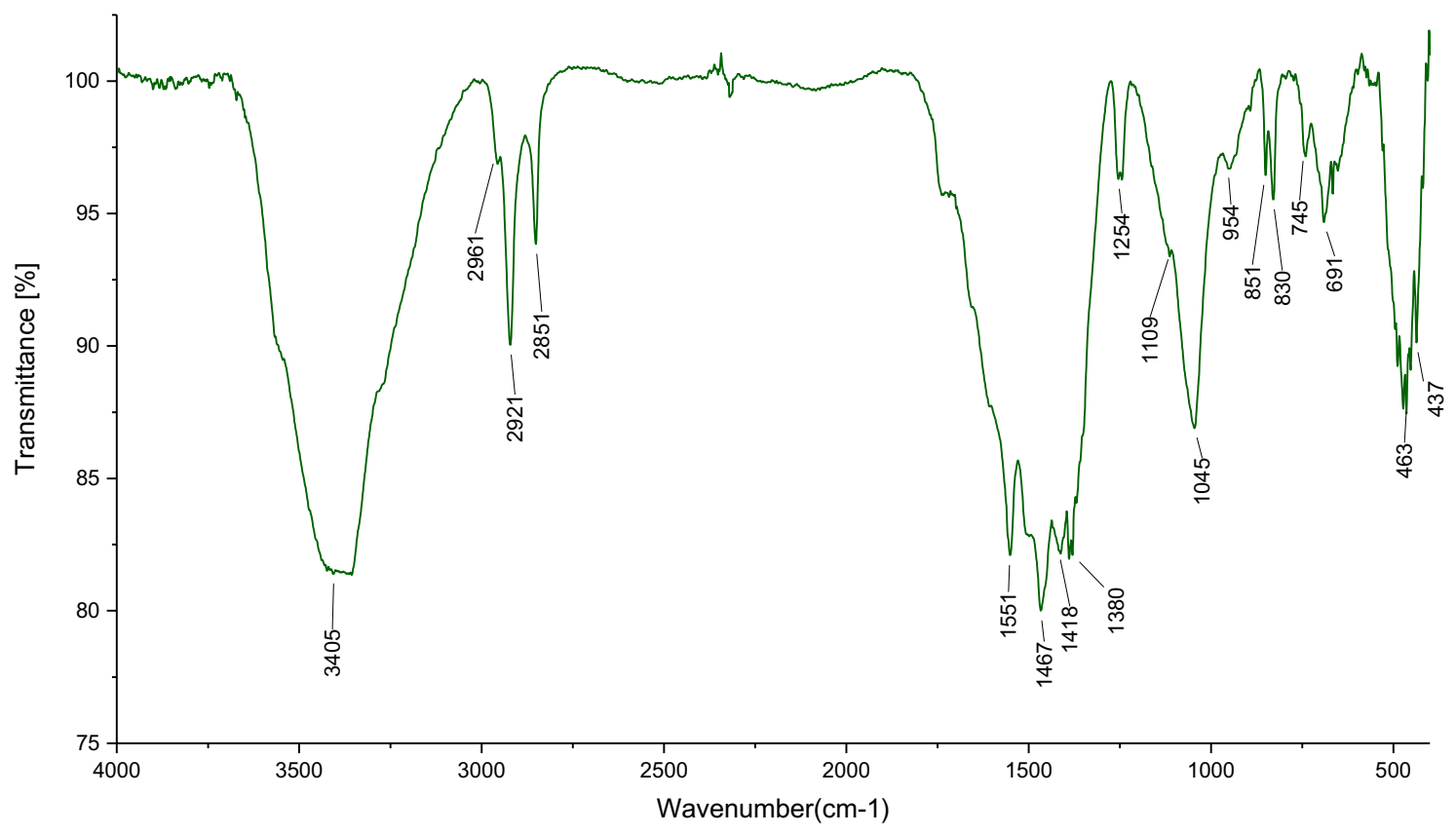

Figure 10. FT-IR Spectrum of spent nanoporous Zn-MOF catalyst.

\begin{tabular}{|l|l|l|l|l|}
\hline Entry & Catalyst & Time (min) & Yield (\%) & Ref. \\
\hline 1 & $\mathrm{FeAl}_{2} \mathrm{O}_{4}$ & 180 & 90 & 52 \\
\hline 2 & $\mathrm{Fe}_{3} \mathrm{O}_{4} @ \mathrm{D}-\mathrm{NH}-\left(\mathrm{CH}_{2}\right)_{4}-\mathrm{SO}_{3} \mathrm{H}$ & 90 & 86 & 53 \\
\hline 3 & $\mathrm{Fe}_{3} \mathrm{O}_{4} @ \mathrm{FSM}-16-\mathrm{SO}_{3} \mathrm{H}$ & 25 & 86 & 54 \\
\hline 4 & $\mathrm{Fe}_{3} \mathrm{O}_{4}-\mathrm{TEDETA}-\mathrm{Br}_{3}$ & 120 & 92 & 55 \\
\hline 5 & $\left.\mathrm{Fe}_{3} \mathrm{O}_{4} @ \mathrm{SiO}_{2} @\left(\mathrm{CH}_{2}\right)_{3} \mathrm{Im}\right\} \mathrm{C}\left(\mathrm{NO}_{2}\right)_{3}$ & 18 & 89 & 56 \\
\hline 6 & $\mathrm{Fe}_{3} \mathrm{O}_{4} @ \mathrm{SiO}_{2}-\mathrm{PEG} / \mathrm{NH}_{2}$ & 20 & 96 & 57 \\
\hline 7 & $\mathrm{Fe}_{3} \mathrm{O}_{4} @ \mathrm{GA} @ \mathrm{IG}$ & 45 & 89 & 41 \\
\hline 8 & $\mathrm{AIL}-\mathrm{SCMNPs}$ & 15 & 80 & 58 \\
\hline 9 & $\mathrm{Zn}-\mathrm{MOF}$ & 85 & 96 & This work \\
\hline
\end{tabular}

Table 3. Comparison of the polyhydroquinolines synthesis in presence of various catalysts. ${ }^{\text {a }}$ Isolated yield.

Received: 4 October 2021; Accepted: 12 January 2022

Published online: 27 January 2022

\section{References}

1. Xu, J. et al. Three-dimensional walnut-like, hierarchically nanoporous carbon microspheres: One-pot synthesis, activation, and supercapacitive performance. ACS Sustain. Chem. Eng. 8, 8024-8036 (2020).

2. Cui, W.-G., Zhang, G.-Y., Hu, T.-L. \& Bu, X.-H. Metal-organic framework-based heterogeneous catalysts for the conversion of C1 chemistry: CO, CO2 and CH4. Coord. Chem. Rev. 387, 79-120 (2019).

3. Cui, W.-G. et al. Engineering $\mathrm{Co} / \mathrm{MnO}$ heterointerface inside porous graphitic carbon for boosting the low-temperature $\mathrm{CO} 2 \mathrm{metha}-$ nation. Appl. Catal. B Environ. 287, 119959 (2021).

4. Yu, D. et al. A solvent-assisted ligand exchange approach enables metal-organic frameworks with diverse and complex architectures. Nat. Commun. 11, 927 (2020).

5. Ahmad, N. et al. Development of mixed metal metal-organic polyhedra networks, colloids, and MOFs and their pharmacokinetic applications. Sci. Rep. 7, 832 (2017).

6. Supercapacitor made from MOF. Nature 538, 143 (2016).

7. Li, T., Bai, Y., Wang, Y., Xu, H. \& Jin, H. Advances in transition-metal (Zn, Mn, Cu)-based MOFs and their derivatives for anode of lithium-ion batteries. Coord. Chem. Rev. 410, 213221 (2020).

8. Mohamed, N. A. et al. Studies on metal-organic framework (MOF) nanomedicine preparations of sildenafil for the future treatment of pulmonary arterial hypertension. Sci. Rep. 11, 4336 (2021).

9. Su, Y.-S. et al. Dipolar order in an amphidynamic crystalline metal-organic framework through reorienting linkers. Nat. Chem. 13, 278-283 (2021).

10. Koolivand, M., Nikoorazm, M., Ghorbani-Choghamarani, A. \& Tahmasbi, B. Cu-citric acid metal-organic framework: Synthesis, characterization and catalytic application in Suzuki-Miyaura cross-coupling reaction and oxidation of sulfides. Appl. Organomet. Chem. 1, e6434 (2021).

11. Ghorbani-Choghamarani, A., Taherinia, Z. \& Mohammadi, M. Facile synthesis of Fe3O4@GlcA@Ni-MOF composites as environmentally green catalyst in organic reactions. Environ. Technol. Innov. 1, 102050. https://doi.org/10.1016/j.eti.2021.102050 (2021). 
12. Rogowski, L. W. et al. Symmetry breaking propulsion of magnetic microspheres in nonlinearly viscoelastic fluids. Nat. Commun. 12, $1116(2021)$.

13. Hu, Y. et al. Bis(zinc(II)-dipicolylamine)-functionalized sub-2 $\mu \mathrm{m}$ core-shell microspheres for the analysis of $\mathrm{N}$-phosphoproteome. Nat. Commun. 11, 6226 (2020).

14. Ding, Y. et al. Preparation of hollow $\mathrm{Cu}$ and $\mathrm{CuOx}$ microspheres with a hierarchical structure for heterogeneous catalysis. ACS Appl. Mater. Interfaces 10, 41793-41801 (2018).

15. Feng, Y. et al. Sea-urchin-like hollow CuMoO4-CoMoO4 hybrid microspheres, a noble-metal-like robust catalyst for the fast hydrogen production from ammonia borane. ACS Appl. Energy Mater. 4, 633-642 (2021).

16. Wu, J. et al. One-step preparation and characterization of silk nano- and microspheres. Polym. J. 52, 1395-1400 (2020).

17. GhoshDastidar, D., Saha, S. \& Chowdhury, M. Porous microspheres: Synthesis, characterisation and applications in pharmaceutical \& medical fields. Int. J. Pharm. 548, 34-48 (2018).

18. Kumar, G. P. \& Anand, J. Porous floating microspheres: A new dimension in controlled drug delivery. Res. J. Pharm. Technol. 4, 1340-1357 (2011).

19. Molvinger, K., Quignard, F., Brunel, D., Boissière, M. \& Devoisselle, J.-M. Porous chitosan-silica hybrid microspheres as a potential catalyst. Chem. Mater. 16, 3367-3372 (2004).

20. Ahmed, A., Skinley, K., Herodotou, S. \& Zhang, H. Core-shell microspheres with porous nanostructured shells for liquid chromatography. J. Sep. Sci. 41, 99-124 (2018).

21. He, J.-H., Xu, L., Wu, Y. \& Liu, Y. Mathematical models for continuous electrospun nanofibers and electrospun nanoporous microspheres. Polym. Int. 56, 1323-1329 (2007).

22. Na, X., Zhou, W., Li, J., Su, Z. \& Ma, G. Preparation and application of porous polymer microspheres in virus-like particles purification. Prog. Chem. 30, 5-13 (2018).

23. Xu, J. et al. One-stage synthesis of cagelike porous polymeric microspheres and application as catalyst scaffold of Pd nanoparticles. Macromolecules 44, 3730-3738 (2011).

24. Hamaloğlu, K. Ö. et al. Monodisperse-porous cerium oxide microspheres carrying iridium oxide nanoparticles as a heterogeneous catalyst for water oxidation. Appl. Surf. Sci. 547, 149219 (2021).

25. Yang, S. et al. Zinc-iron bimetallic-nitrogen doped porous carbon microspheres as efficient oxygen reduction electrocatalyst for zinc-air batteries. Appl. Surf. Sci. 546, 148934 (2021).

26. Wang, T., Cui, G., Zhao, Y., Nurpeissova, A. \& Bakenov, Z. Porous carbon nanotubes microspheres decorated with strong catalyst cobalt nanoparticles as an effective sulfur host for lithium-sulfur battery. J. Alloys Compd. 853, 157268 (2021).

27. Hamaloğlu, K. Ö. et al. Monodisperse-porous cerium oxide microspheres as a new support with appreciable catalytic activity for a composite catalyst in benzyl alcohol oxidation. New J. Chem. 45, 2019-2029 (2021).

28. Hasani, A. et al. Recent progress of two-dimensional materials and metal-organic framework-based taste sensors. J. Korean Ceram. Soc. 57, 353-367 (2020).

29. Chen, D., Wei, L., Li, J. \& Wu, Q. Nanoporous materials derived from metal-organic framework for supercapacitor application. J. Energy Storage 30, 101525 (2020).

30. Gong, Y., Zhu, Z., Qian, Q., Tong, W. \& Gong, H. Zn- And Cu-catalyzed coupling of tertiary alkyl bromides and oxalates to forge challenging C-O, C-S, and C-N bonds. Org. Lett. 23, 1005-1010 (2021).

31. Shen, H.-M. et al. Efficient oxidation of cycloalkanes with simultaneously increased conversion and selectivity using $\mathrm{O} 2$ catalyzed by metalloporphyrins and boosted by $\mathrm{Zn}(\mathrm{AcO}) 2$ : A practical strategy to inhibit the formation of aliphatic diacids. Appl. Catal. A Gen. 609, 117904 (2021).

32. Trost, B. M. \& Zhu, C. Zn-ProPhenol catalyzed enantioselective mannich reaction of $2 \mathrm{H}$-azirines with alkynyl ketones. Org. Lett. 22, 9683-9687 (2020).

33. Yuan, Y. et al. Synthesis of aza-eight-membered ring-fused indolines initiated by Zn-catalyzed C2 alkylation of indoles and subsequent base-promoted ring expansion. Org. Lett. 22, 6532-6536 (2020).

34. Trost, B. M. et al. Direct enantio- and diastereoselective Zn-prophenol-catalyzed mannich reactions of CF 3 - and SCF 3 -substituted ketones. Org. Lett. 22, 2437-2441 (2020).

35. Mohammadi, M., Khodamorady, M., Tahmasbi, B., Bahrami, K. \& Ghorbani-Choghamarani, A. Boehmite nanoparticles as versatile support for organic-inorganic hybrid materials: Synthesis, functionalization, and applications in eco-friendly catalysis. J. Ind. Eng. Chem. https://doi.org/10.1016/j.jiec.2021.02.001 (2021).

36. Kazemi, M. \& Mohammadi, M. Magnetically recoverable catalysts: Catalysis in synthesis of polyhydroquinolines. Appl. Organomet. Chem. 34, e5400 (2020).

37. Evans, C. G. \& Gestwicki, J. E. Enantioselective organocatalytic hantzsch synthesis of polyhydroquinolines. Org. Lett. 11, 2957-2959 (2009).

38. Rathee, G., Kohli, S., Singh, N., Awasthi, A. \& Chandra, R. Calcined layered double hydroxides: Catalysts for xanthene, 1,4-dihydropyridine, and polyhydroquinoline derivative synthesis. ACS Omega 5, 15673-15680 (2020).

39. Rathee, G. et al. Fabrication of a gold-supported NiAlTi-layered double hydroxide nanocatalyst for organic transformations. ACS Omega 5, 23967-23974 (2020).

40. Sashidhara, K. V. et al. Discovery of coumarin-dihydropyridine hybrids as bone anabolic agents. J. Med. Chem. 56, 109-122 (2013).

41. Pourian, E., Javanshir, S., Dolatkhah, Z., Molaei, S. \& Maleki, A. Ultrasonic-assisted preparation, characterization, and use of novel biocompatible core/shell Fe3O4@GA@Isinglass in the synthesis of 1,4-dihydropyridine and 4 H-pyran derivatives. ACS Omega 3, 5012-5020 (2018).

42. Tamoradi, T., Mohammadi, M., Kiasat, A. R., Davarpanah, J. \& Karmakar, B. A competent, atom-efficient and sustainable synthesis of bis-coumarin derivatives catalyzed over strontium-doped asparagine modified graphene oxide nanocomposite. Polycycl. Aromat. Compd. 1, 1-15 (2021).

43. Sam, M., Dekamin, M. G. \& Alirezvani, Z. Dendrons containing boric acid and 1,3,5-tris(2-hydroxyethyl)isocyanurate covalently attached to silica-coated magnetite for the expeditious synthesis of Hantzsch esters. Sci. Rep. 11, 2399. https://doi.org/10.1080/ 10406638.2021.1998149 (2021).

44. Moavi, J., Buazar, F. \& Sayahi, M. H. Algal magnetic nickel oxide nanocatalyst in accelerated synthesis of pyridopyrimidine derivatives. Sci. Rep. 11, 6296 (2021).

45. Yang, M. \& Bai, Q. Flower-like hierarchical Ni-Zn MOF microspheres: Efficient adsorbents for dye removal. Colloids Surf. A. 582, 123795 (2019).

46. Lupan, O., Chow, L., Chai, G. \& Heinrich, H. Fabrication and characterization of $\mathrm{Zn}-\mathrm{ZnO}$ core-shell microspheres from nanorods. Chem. Phys. Lett. 465, 249-253 (2008).

47. Maleki, B., Reiser, O., Esmaeilnezhad, E. \& Choi, H. J. SO3H-dendrimer functionalized magnetic nanoparticles (Fe3O4@D-NH(CH2)4-SO3H): Synthesis, characterization and its application as a novel and heterogeneous catalyst for the one-pot synthesis of polyfunctionalized pyrans and polyhydroquinolines. Polyhedron 162, 129-141 (2019).

48. Moradi, L. \& Tadayon, M. Green synthesis of 3,4-dihydropyrimidinones using nano Fe3O4@meglumine sulfonic acid as a new efficient solid acid catalyst under microwave irradiation. J. Saudi Chem. Soc. 22, 66-75 (2018).

49. Ahmadi, A., Sedaghat, T., Azadi, R. \& Motamedi, H. Magnetic mesoporous silica nanocomposite functionalized with palladium schiff base complex: Synthesis, characterization, catalytic efficacy in the suzuki-miyaura reaction and $\alpha$-amylase immobilization. Catal. Lett. 150, 112-126 (2020). 
50. Amoozadeh, A., Rahmani, S., Bitaraf, M., Abadi, F. B. \& Tabrizian, E. Nano-zirconia as an excellent nano support for immobilization of sulfonic acid: a new, efficient and highly recyclable heterogeneous solid acid nanocatalyst for multicomponent reactions. New J. Chem. 40, 770-780 (2016).

51. Ghorbani-Choghamarani, A., Mohammadi, M., Tamoradi, T. \& Ghadermazi, M. Covalent immobilization of Co complex on the surface of SBA-15: Green, novel and efficient catalyst for the oxidation of sulfides and synthesis of polyhydroquinoline derivatives in green condition. Polyhedron 158, 25-35 (2019).

52. Ghorbani-Choghamarani, A., Mohammadi, M., Shiri, L. \& Taherinia, Z. Synthesis and characterization of spinel FeAl2O4 (hercynite) magnetic nanoparticles and their application in multicomponent reactions. Res. Chem. Intermed. 45, 5705-5723 (2019).

53. Alinezhad, H., Tarahomi, M., Maleki, B. \& Amiri, A. SO 3 H-functionalized nano-MGO-D-NH 2: Synthesis, characterization and application for one-pot synthesis of pyrano[2,3-d]pyrimidinone and tetrahydrobenzo[b]pyran derivatives in aqueous media. Appl. Organomet. Chem. 33, 1-10 (2019).

54. Hashemi-Uderji, S., Abdollahi-Alibeik, M. \& Ranjbar-Karimi, R. Fe 3 O 4 @FSM-16-SO 3 H as a novel magnetically recoverable nanostructured catalyst: Preparation, characterization and catalytic application. J. Porous Mater. 26, 467-480 (2019).

55. Hajjami, M. \& Gholamian, F. Tribromide ion immobilized on magnetic nanoparticle as a new, efficient and reusable nanocatalyst in multicomponent reactions. RSC Adv. 6, 87950-87960 (2016).

56. Yarie, M. et al. Novel magnetic nanoparticles with ionic liquid tags as a reusable catalyst in the synthesis of polyhydroquinolines. RSC Adv. 6, 82842-82853 (2016).

57. Kardooni, R., Kiasat, A. R. \& Motamedi, H. Designing of a novel dual-function silica-iron oxide hybrid based nanocomposite, Fe3O4@SiO2[sbnd]PEG/NH2, and its application as an eco-catalyst for the solvent-free synthesis of polyhydroacridines and polyhydroquinolines. J. Taiwan Inst. Chem. Eng. 81, 373-382 (2017).

58. Taheri, N., Heidarizadeh, F. \& Kiasat, A. A new magnetically recoverable catalyst promoting the synthesis of 1,4-dihydropyridine and polyhydroquinoline derivatives via the Hantzsch condensation under solvent-free conditions. J. Magn. Magn. Mater. 428, 481-487 (2017).

\section{Acknowledgements}

This work was supported by the research facilities of Ilam University, Ilam, Iran, and Bu-Ali Sina University, Hamedan, Iran.

\section{Author contributions}

S.M.R. did the experimental works. A.G-C. supervised the research project and is the corresponding authorof the manuscript. M.M. write the manuscript draf.

\section{Competing interests}

The authors declare no competing interests.

Additional information

Supplementary Information The online version contains supplementary material available at https://doi.org/ 10.1038/s41598-022-05411-8.

Correspondence and requests for materials should be addressed to A.G.-C.

Reprints and permissions information is available at www.nature.com/reprints.

Publisher's note Springer Nature remains neutral with regard to jurisdictional claims in published maps and institutional affiliations.

(c) (1) Open Access This article is licensed under a Creative Commons Attribution 4.0 International License, which permits use, sharing, adaptation, distribution and reproduction in any medium or format, as long as you give appropriate credit to the original author(s) and the source, provide a link to the Creative Commons licence, and indicate if changes were made. The images or other third party material in this article are included in the article's Creative Commons licence, unless indicated otherwise in a credit line to the material. If material is not included in the article's Creative Commons licence and your intended use is not permitted by statutory regulation or exceeds the permitted use, you will need to obtain permission directly from the copyright holder. To view a copy of this licence, visit http://creativecommons.org/licenses/by/4.0/.

(C) The Author(s) 2022 